\title{
Insect pest resistance: an alternative approach for crop protection
}

\begin{abstract}
From experience with insect resistance caused by synthetic chemical insecticides, it is clear that no single management tactic can provide lasting solutions to the insect pest problem. Biological control is a component of integrated pest management strategies that minimize insecticide spray applications and move towards ecofriendly systems of pest management. Successful utilization of host plant resistance, phytochemical products, pheromones, biological control agents such as predators, parasitoids, entomopathogenic bacteria, virus, nematodes, and fungi can help to control many destructive pests to achieve sustainable crop protection.
\end{abstract}

Keyword: Insecticide; Resistance; Biological control; Biopesticides 